\title{
Dynamics of water eutrophication model with control
}

\author{
Jianjun Jiao ${ }^{1 *}$, Pei Li ${ }^{1}$ and Dahe Feng ${ }^{1}$
}

\section{"Correspondence:}

jiaojianjun05@126.com

1 School of Mathematics and

Statistics, Guizhou University of

Finance and Economics, Guiyang,

P.R. China

\begin{abstract}
In this work, we consider a water eutrophication model with impulsive dredging. We prove that all solutions of the investigated system are uniformly bounded. There exists globally asymptotically stable periodic solution of microorganism-extinction when some condition is satisfied. The condition for permanence of the system is also obtained. It is concluded that the approach of impulsive dredging provides a reliable theoretical basis for the management of water eutrophication.
\end{abstract}

Keywords: Impulsive dredging; Water eutrophication; Extinction; Permanence

\section{Introduction}

Due to human activities, there are more and more nutrients in water, which causes excessive reproduction of algae and other aquatic organisms, decrease in water transparency, dissolved oxygen reduction, deterioration of water quality, and so on. This kind of pollution phenomenon, which is caused by the change in the ecological balance of the whole water body, is called the eutrophication of water body [1].

Eutrophication occurs in ponds, reservoirs, lakes, and so on and it has attracted the attention of many experts and scholars in the early twentieth century. Especially in recent years, the study on the eutrophication of water body has become quite active like fuzzy mathematics, stochastic model, gray system, artificial intelligence, and other theoretical methods combined with computer technology [2-4]. For instance, based on the analysis of the present situation and causes of pollution in Chaohu, Li et al. [5] proposed specific measures for eutrophication control of Chaohu. Yu et al. [6] used three methodsCarlson comprehensive index method, Grey clustering method, and principal component analysis - to analyze the main factors of eutrophication, and they provided theoretical basis for controlling water pollution in Poyang Lake. In addition, many scholars [7-9] have studied the dynamics model of a single population in the polluted environment.

Many scholars have done in-depth research on the problems of eutrophication. But most of them studied the reasons and the prevention and treatment of water eutrophication from the technical aspects. Few researchers studied the problem of eutrophication from the viewpoint of biological dynamics. In this paper, we discuss water eutrophication with impulsive dredging.

(c) The Author(s) 2018. This article is distributed under the terms of the Creative Commons Attribution 4.0 International License (http://creativecommons.org/licenses/by/4.0/), which permits unrestricted use, distribution, and reproduction in any medium, provided you give appropriate credit to the original author(s) and the source, provide a link to the Creative Commons license, and indicate if changes were made. 


\section{Model formulation}

In this paper, we consider the following eutrophication model with control:

$$
\left\{\begin{array}{l}
\left.\begin{array}{l}
\frac{d s(t)}{d t}=\lambda-d_{1} s(t)-\beta s(t) x(t) \\
\frac{d x(t)}{d t}=\frac{\beta}{\delta} s(t) x(t)-d_{2} x(t)
\end{array}\right\} \quad t \neq n \tau, t \neq(n+l) \tau, \\
\Delta s(t)=\mu \\
\Delta x(t)=0 \\
\Delta s(t)=-\mu_{1} s(t) \\
\Delta x(t)=-\mu_{2} x(t)
\end{array}\right\} \quad t=(n+l) \tau, 0<l<1, n=1,2, \ldots,
$$

where $s(t)$ denotes the concentration of nutrients in water. $x(t)$ denotes the concentration of microorganism in water. $\lambda>0$ is the original concentration of nutrients $s(t)$ in water, $\beta>0$ is called the transmission coefficient. $\frac{\beta}{\delta}>0$ is called the transmission of $x(t)$ absorbed nutrients. $d_{1}>0$ is called the loss of $s(t) . d_{2}>0$ is called the death coefficient of $x(t) . \mu>0$ is the increase amount of $s(t)$ at $t=n \tau .0 \leq \mu_{1}<1,0 \leq \mu_{2}<1$ respectively represent the dredging intensity of $s(t)$ and $x(t)$ at $t=(n+l) \tau(0<l<1), \tau$ is the period of the impulsive effect.

\section{Analyzing model}

Let $R_{+}=[0, \infty)$, The solution of system (2.1), denoted by $y(t)=(s(t), x(t))^{T}$, is a piecewise continuous function $y: R_{+} \rightarrow R_{+}^{2}, y(t)$ is continuous on $(n \tau,(n+l) \tau],((n+l) \tau,(n+1) \tau]$, $n \in Z_{+}$and $y\left(n \tau^{+}\right)=\lim _{t \rightarrow n \tau^{+}} y(n \tau), y\left((n+l) \tau^{+}\right)=\lim _{t \rightarrow(n+l) \tau^{+}} y((n+l) \tau)$ exist. Obviously the global existence and uniqueness of the solutions of (2.1) are guaranteed by the smoothness of function $y$, which denotes the mapping defined by the right-hand side of system (2.1) (see [10]). Before discussing the main results, we will give some definitions, notations, and lemmas. Since $s(t)=0$ whenever $s^{\prime}(t)=\lambda, x(t)=0$ whenever $x^{\prime}(t)=0$, and $s\left(n \tau^{+}\right)=s(n \tau)+\mu, \mu \geq 0$, so we have the following.

Lemma 1 Suppose that $y(t)$ is a solution of $(2.1)$, with $y\left(0^{+}\right) \geq 0$, then $y(t) \geq 0$ for $t \geq 0$ and further $y(t)>0, t \geq 0$ for $y\left(0^{+}\right)>0$.

Lemma 2 (see [10, p. 23, Lemma 2.2]) Let the function $m \in P C^{\prime}\left[R^{+}, R\right]$ satisfy the inequalities

$$
\begin{cases}m^{\prime}(t) \leq p(t) m(t)+q(t), & t \geq t_{0}, t \neq t_{k}, k=1,2, \ldots \\ m\left(t_{k}^{+}\right) \leq d_{k} m\left(t_{k}\right)+b_{k}, & t=t_{k}\end{cases}
$$

where $p, q \in P C\left[R^{+}, R\right], d_{k} \geq 0, b_{k}$ are constants, then

$$
\begin{aligned}
m(t) \leq & m\left(t_{0}\right) \prod_{t_{0}<t_{k}<t} d_{k} \exp \left(\int_{t_{0}}^{t} p(s) d s\right)+\sum_{t_{0}<t_{k}<t}\left(\prod_{t_{0}<t_{k}<t} d_{j} \exp \left(\int_{t_{0}}^{t} p(s) d s\right)\right) b_{k} \\
& +\int_{t_{0}}^{t} \prod_{s<t_{k}<t} d_{k} \exp \left(\int_{s}^{t} p(\sigma) d \sigma\right) q(s) d s, \quad t \geq t_{0} .
\end{aligned}
$$

Now, we show that all solutions of (2.1) are uniformly ultimately bounded. 
Lemma 3 There exists a constant $M>0$ such that $S(t) \leq M, x(t) \leq M$ for each solution $(s(t), x(t))$ of $(2.1)$ with all t large enough.

Proof Definition $V(t)=\frac{1}{\delta} s(t)+x(t), d=\min \left\{d_{1}, d_{2}\right\}$. Then $t \neq n \tau, t \neq(n+l) \tau$. We have

$$
\begin{aligned}
D^{+} V(t)+d V(t) & =\frac{1}{\delta}\left(\lambda-d_{1} s(t)-\beta s(t) x(t)\right)+\frac{\beta}{\delta} s(t) x(t)-d_{2} x(t)+\frac{d}{\delta} s(t)+d x(t) \\
& =\frac{\lambda}{\delta}-\frac{d_{1}-d}{\delta} s(t)-\left(d_{2}-d\right) x(t) \\
& \leq \frac{\lambda}{\delta} .
\end{aligned}
$$

When $t=n \tau$,

$$
V\left(n \tau^{+}\right)=\frac{1}{\delta} s\left(n \tau^{+}\right)+x\left(n \tau^{+}\right)=\frac{1}{\delta}(s(n \tau)+\mu)+x(n \tau)=\frac{1}{\delta} s(n \tau)+x(n \tau)+\frac{\mu}{\delta}=V(n \tau)+\frac{\mu}{\delta} .
$$

When $t=(n+l) \tau$,

$$
\begin{aligned}
V\left((n+l) \tau^{+}\right) & =\frac{1}{\delta} s\left((n+l) \tau^{+}\right)+x\left((n+l) \tau^{+}\right) \\
& =\frac{1}{\delta}\left(1-\mu_{1}\right) s((n+l) \tau)+\left(1-\mu_{2}\right) x((n+l) \tau) \leq V((n+l) \tau) .
\end{aligned}
$$

By Lemma 2, for $t \in(n \tau, n+l) \tau], t \in((n+l) \tau,(n+1) \tau]$, we have

$$
\begin{cases}D^{+} V(t) \leq-d V(t)+\frac{\lambda}{\delta}, & t \neq n \tau, t \neq(n+l) \tau \\ V\left(t^{+}\right)=V(t)+\frac{\mu}{\delta}, & t=n \tau \\ V\left(t^{+}\right) \leq V(t), & t=(n+l) \tau\end{cases}
$$

then

$$
\begin{aligned}
V(t) & \leq V(0) \exp (-d t)+\int_{0}^{t} \frac{\lambda}{\delta} \exp (-d(t-s)) d s+\sum_{0<n \tau<t} \frac{\mu}{\delta} \exp (-d(t-n \tau)) \\
& \rightarrow \frac{\lambda}{\delta d}+\frac{\mu \exp (d \tau)}{\delta(\exp (d \tau)-1)}, \quad \text { as } t \rightarrow \infty .
\end{aligned}
$$

So $V(t)$ is uniformly ultimately bounded. Hence, by the definition of $V(t)$, there exists a constant $M>0$ such that $S(t) \leq M, x(t) \leq M$ for $t$ large enough. The proof is complete.

If $x(t)=0$, we have the following subsystem of (3.1):

$$
\begin{cases}s^{\prime}(t)=\lambda-d_{1} s(t), & t \neq n \tau, t \neq(n+l) \tau \\ \Delta s(t)=\mu, & t=n \tau, \\ \Delta s(t)=-\mu_{1} s(t), & t=(n+l) \tau \\ s\left(0^{+}\right)=s(0)>0 . & \end{cases}
$$

We can easily obtain the analytic solution of (3.1) between pulses as follows:

$$
s(t)= \begin{cases}\frac{1}{d_{1}}\left[\lambda-\left(\lambda-d_{1} s\left(n \tau^{+}\right)\right) e^{-d_{1}(t-n \tau)}\right], & t \in(n \tau,(n+l) \tau], \\ \frac{1}{d_{1}}\left[\lambda-\left(\lambda-d_{1} s\left((n+l) \tau^{+}\right)\right) e^{-d_{1}(t-(n+l) \tau)}\right], & t \in((n+l) \tau,(n+1) \tau] .\end{cases}
$$


Considering the third equation of (3.1), we have

$$
s\left((n+l) \tau^{+}\right)=\frac{1-\mu_{1}}{d_{1}}\left[\lambda-\left(\lambda-d_{1} s\left(n \tau^{+}\right)\right) e^{-d_{1} l \tau}\right] .
$$

Considering the fifth equation of (3.1), we also have

$$
s\left((n+1) \tau^{+}\right)=\frac{1}{d_{1}}\left[\lambda-\left(\lambda-d_{1} s\left((n+l) \tau^{+}\right)\right) e^{-d_{1}(1-l) \tau}\right]+\mu .
$$

Substituting (3.3) into (3.4), we have

$$
\begin{aligned}
s\left((n+1) \tau^{+}\right)= & \frac{\lambda}{d_{1}}-\frac{\lambda e^{-d_{1}(1-l) \tau}}{d_{1}}+\frac{\lambda\left(1-\mu_{1}\right) e^{-d_{1}(1-l) \tau}}{d_{1}} \\
& -\frac{\lambda\left(1-\mu_{1}\right) e^{-d_{1} \tau}}{d_{1}}+\left(1-\mu_{1}\right) s\left(n \tau^{+}\right) e^{-d_{1} \tau}+\mu .
\end{aligned}
$$

Equation (3.1) has one fixed point as follows:

$$
s^{*}=\frac{\lambda-\lambda \mu_{1} e^{-d_{1}(1-l) \tau}-\lambda\left(1-\mu_{1}\right) e^{-d_{1} \tau}+\mu d_{1}}{d_{1}\left[1-\left(1-\mu_{1}\right) e^{-d_{1} \tau}\right]} .
$$

Then the periodic solution of (3.1) is

$$
\tilde{s}(t)= \begin{cases}\frac{1}{d_{1}}\left[\lambda-\left(\lambda-d_{1} s^{*}\right) e^{-d_{1}(t-n \tau)}\right], & t \in[n \tau,(n+l) \tau), \\ \frac{1}{d_{1}}\left[\lambda-\left(\lambda-d_{1} s^{* *}\right) e^{-d_{1}(t-(n+l) \tau)}\right], & t \in[(n+l) \tau,(n+1) \tau) .\end{cases}
$$

Here $s^{*}$ is determined as (3.6), $s^{* *}$ is defined as

$$
s^{* *}=\frac{1-\mu_{1}}{d_{1}}\left[\lambda-\left(\lambda-d_{1} s^{*}\right) e^{-d_{1} l \tau}+d_{1}\left(1-\mu_{1}\right) s^{*} e^{-d_{1} l \tau}\right] .
$$

It is similar to reference [8], we can obtain the following lemma.

Lemma 4 System (3.1) has a positive periodic solution $\tilde{s}(t)$. For every solution $s(t)$ of system (3.1), we have $s(t) \rightarrow \tilde{s}(t)$ as $t \rightarrow \infty$.

\section{The dynamics}

From the above discussion, we know that system (2.1) has a microorganism-extinction periodic solution $(\tilde{s}(t), 0)$. So we can have the following theorem.

\section{Theorem 1 If}

$$
\ln \frac{1}{1-\mu_{2}}>\frac{\lambda \tau}{d_{1}}-\frac{\lambda-d_{1} s^{*}}{d_{1}^{2}}\left(1-e^{-d_{1} l \tau}\right)-\frac{\lambda-d_{1} s^{* *}}{d_{1}^{2}}\left(1-e^{-d_{1}(1-l) \tau}\right) e^{-d_{1} \tau}-d_{2} \tau
$$

holds, then the microorganism-extinction periodic solution $(\tilde{s}(t), 0)$ of system (2.1) is globally asymptotically stable. 
Proof First we prove the local stability. Defining $S(t)=s(t)-\tilde{s}(t), X(t)=x(t)$, we have the following linearly similar system for $(2.1)$ which concerns one periodic solution $(\tilde{s}(t), 0)$ :

$$
\left(\begin{array}{c}
S^{\prime}(t) \\
X^{\prime}(t)
\end{array}\right)=\left(\begin{array}{cc}
-d_{1} & \beta \tilde{s}(t) \\
0 & \frac{\beta}{\delta} \tilde{s}(t)-d_{2}
\end{array}\right)\left(\begin{array}{c}
S(t) \\
X(t)
\end{array}\right)
$$

It is easy to obtain the fundamental solution matrix

$$
\Phi(t)=\left(\begin{array}{cc}
e^{-d_{1} t} & * \\
0 & \exp \left(\int_{0}^{t}\left(\frac{\beta}{\delta} \tilde{s}(s)-d_{2}\right) d s\right)
\end{array}\right) .
$$

There is no need to calculate the exact form of $*$ as it is not required in the analysis that follows. The linearization of the third and fourth equations of (2.1) is as follows:

$$
\left(\begin{array}{l}
S\left(n \tau^{+}\right) \\
X\left(n \tau^{+}\right)
\end{array}\right)=\left(\begin{array}{ll}
1 & 0 \\
0 & 1
\end{array}\right)\left(\begin{array}{l}
S(n \tau) \\
X(n \tau)
\end{array}\right) .
$$

The linearization of the fifth and sixth equations of (2.1) is as follows:

$$
\left(\begin{array}{l}
S\left((n+l) \tau^{+}\right) \\
X\left((n+l) \tau^{+}\right)
\end{array}\right)=\left(\begin{array}{cc}
1-\mu_{1} & 0 \\
0 & 1-\mu_{2}
\end{array}\right)\left(\begin{array}{l}
S((n+l) \tau) \\
X((n+l) \tau)
\end{array}\right) .
$$

The stability of the periodic solution $(\tilde{s}(t), 0)$ is determined by the eigenvalues of

$$
M=\left(\begin{array}{ll}
1 & 0 \\
0 & 1
\end{array}\right)\left(\begin{array}{cc}
1-\mu_{1} & 0 \\
0 & 1-\mu_{2}
\end{array}\right) \phi(\tau)
$$

which are

$$
\lambda_{1}=\left(1-\mu_{1}\right) e^{-d_{1} t}<1, \quad \lambda_{2}=\left(1-\mu_{2}\right) \exp \left(\int_{0}^{\tau}\left(\frac{\beta}{\delta} \tilde{s}(s)-d_{2}\right) d s\right) .
$$

According to the Floquet theory [11], if $\left|\lambda_{2}\right|<1$, i.e.,

$$
\left|\left(1-\mu_{2}\right) \exp \left(\int_{0}^{\tau}\left(\frac{\beta}{\delta} \tilde{s}(t)-d_{2}\right) d t\right)\right|<1,
$$

that is,

$$
\ln \frac{1}{1-\mu_{2}}>\frac{\lambda \tau}{d_{1}}-\frac{\lambda-d_{1} s^{*}}{d_{1}^{2}}\left(1-e^{-d_{1} l \tau}\right)-\frac{\lambda-d_{1} s^{* *}}{d_{1}^{2}}\left(1-e^{-d_{1}(1-l) \tau}\right) e^{-d_{1} \tau}-d_{2} \tau
$$

holds, then $(\tilde{s}(t), 0)$ is locally stable.

The following work is to prove the global attraction. Choose $\varepsilon>0$ such that

$$
\rho=\left(1-\mu_{2}\right) \exp \left(\int_{0}^{\tau}\left[\frac{\beta}{\delta}(\tilde{s}(s)-\varepsilon)-d_{2}\right] d s\right)<1 .
$$


From the first equation of (2.1), we notice that $\frac{d s(t)}{d t} \leq \lambda-d_{1} s(t)$. So we consider the following impulsive differential equations:

$$
\begin{cases}\frac{d y(t)}{d t}=\lambda-d_{1} y(t), & t \neq n \tau, t \neq(n+l) \tau, \\ \Delta y(t)=\mu, & t=n \tau \\ \Delta y(t)=-\mu_{2} y(t), & t=(n+l) \tau .\end{cases}
$$

From Lemma 2 and the comparison theorem of impulsive differential equations (see Theorem 3.1.1 in [11]), we have $s(t) \leq y(t)$, and, $y(t) \rightarrow \tilde{s}(t)$, as $t \rightarrow \infty$, that is,

$$
s(t) \leq y(t) \leq \tilde{s}(t)+\varepsilon
$$

for all $t$ large enough. For convenience, we may assume (4.3) holds for all $t>0$. From (2.1) and (4.3), we get

$$
\begin{cases}\frac{d x(t)}{d t} \leq \frac{\beta}{\delta}(\tilde{s}(t)+\varepsilon) x(t)-d_{2} x(t), & t \neq n \tau, t \neq(n+l) \tau, \\ \Delta x(t)=0, & t=n \tau, \\ \Delta x(t)=-\mu_{2} x(t), & t=(n+l) \tau .\end{cases}
$$

So

$$
x((n+1) \tau) \leq x\left(n \tau^{+}\right)\left(1-\mu_{2}\right) \exp \left(\int_{n \tau}^{(n+1) \tau}\left[\frac{\beta}{\delta}(\tilde{s}(s)-\varepsilon)-d_{2}\right] d s\right) .
$$

Hence

$$
x((n+l) \tau) \leq x\left(0^{+}\right) \rho^{n}
$$

and $x((n+l) \tau) \rightarrow 0$, as $t \rightarrow \infty$, therefore $x(t) \rightarrow 0$, as $t \rightarrow \infty$.

In what follows, we prove that $s(t) \rightarrow \tilde{s}(t)$, as $t \rightarrow \infty$, let $0<\varepsilon \leq d_{1}$, there must exist $t_{0}>0$ such that $t \geq t_{0}, 0<x(t)<\varepsilon$. Without loss of generality, we assume that $0<x(t)<\varepsilon$ for all $t>0$, then, for system (2.1), we have

$$
\lambda-\left(d_{1}+\varepsilon\right) s(t) \leq \frac{d s(t)}{d t} \leq \lambda-d_{1} s(t)
$$

Then we have $z_{1}(t) \leq s(t) \leq z_{2}(t)$ and $z_{1}(t) \rightarrow \tilde{s}(t), z_{2}(t) \rightarrow \tilde{s}(t)$ as $t \rightarrow \infty$. While $z_{1}(t)$ and $z_{2}(t)$ are the solutions of

$$
\begin{cases}\frac{d z_{1}(t)}{d t}=\lambda-\left(d_{1}+\varepsilon\right) z_{1}(t), & t \neq n \tau, t \neq(n+l) \tau, \\ \Delta z_{1}(t)=\mu, & t=n \tau, \\ \Delta z_{1}(t)=-\mu_{1} z_{1}(t), & t=(n+l) \tau\end{cases}
$$

and

$$
\begin{cases}\frac{d z_{2}(t)}{d t}=\lambda-d_{1} z_{2}(t), & t \neq n \tau, t \neq(n+l) \tau, \\ \Delta z_{2}(t)=\mu, & t=n \tau, \\ \Delta z_{2}(t)=-\mu_{1} z_{2}(t), & t=(n+l) \tau,\end{cases}
$$


respectively

$$
z_{1}(t)= \begin{cases}\frac{1}{d_{1}+\varepsilon}\left[\lambda-\left(\lambda-\left(d_{1}+\varepsilon\right) z_{1}^{*}\right) e^{-\left(d_{1}+\varepsilon\right)(t-n \tau)}\right], & t \in[n \tau,(n+l) \tau), \\ \frac{1}{d_{1}+\varepsilon}\left[\lambda-\left(\lambda-\left(d_{1}+\varepsilon\right) z_{1}^{* *}\right) e^{-\left(d_{1}+\varepsilon\right)(t-(n+l) \tau)}\right], & t \in[(n+l) \tau,(n+1) \tau) .\end{cases}
$$

Here $z_{1}^{*}$ and $z_{1}^{* *}$ are defined as

$$
z_{1}^{*}=\frac{\lambda-\lambda \mu_{1} e^{-\left(d_{1}+\varepsilon\right)(1-l) \tau}-\lambda\left(1-\mu_{1}\right) e^{-\left(d_{1}+\varepsilon\right) \tau}+\left(d_{1}+\varepsilon\right) \mu}{\left(d_{1}+\varepsilon\right)\left[1-\left(1-\mu_{1}\right) e^{-\left(d_{1}+\varepsilon\right) \tau}\right]}
$$

and

$$
z_{1}^{* *}=\frac{\lambda-\mu_{1}}{\left(d_{1}+\varepsilon\right)}\left[\lambda-\left(\lambda-\left(d_{1}+\varepsilon\right) z_{1}^{*}\right) e^{-\left(d_{1}+\varepsilon\right) l \tau}\right] .
$$

Therefore, for any $\varepsilon_{1}>0$, there exists $t_{1}, t>t_{1}$, such that

$$
z_{1}(t)-\varepsilon_{1}<s(t)<\tilde{s}(t)+\varepsilon_{1} .
$$

Let $\varepsilon \rightarrow 0$, so we have

$$
\tilde{s}(t)-\varepsilon_{1}<s(t)<\tilde{s}(t)+\varepsilon_{1}
$$

for $t$ large enough, which implies $s(t) \rightarrow \tilde{s}(t)$ as $t \rightarrow \infty$. This completes the proof.

The following work is to investigate the permanence of system (2.1). Before starting this work, we should give the following definition.

Definition 1 System (2.1) is said to be permanent if there are constants $m, M>0$ (independent of the initial value) and a finite time $T_{0}$ such that for all solutions $(s(t), x(t))$ with all initial values $s\left(0^{+}\right)>0, x\left(0^{+}\right)>0, m \leq s(t) \leq M, m \leq x(t) \leq M$ hold for all $t \geq T_{0}$. Here $T_{0}$ may depend on the initial values $\left(s\left(0^{+}\right), x\left(0^{+}\right)\right)$.

Theorem 2 Let $(s(t), x(t))$ be any solution of system (2.1). If

$$
\ln \frac{1}{1-\mu_{2}}<\frac{\lambda \tau}{d_{1}}-\frac{\lambda-d_{1} s^{*}}{d_{1}^{2}}\left(1-e^{-d_{1} l \tau}\right)-\frac{\lambda-d_{1} s^{* *}}{d_{1}^{2}}\left(1-e^{-d_{1}(1-l) \tau}\right) e^{-d_{1} \tau}-d_{2} \tau
$$

holds, then system (2.1) is permanent.

Proof Let $(s(t), x(t))$ be a solution of (2.1) with $s(0)>0, x(0)>0$. By Lemma 3, we have proved there exists a constant of $M>0$ such that $s(t) \leq M, x(t) \leq M$ for $t$ large enough. We may assume $s(t) \leq M, x(t) \leq M$ for $t \geq 0$. Thus we only need to find $m_{1}>0$ and $\varepsilon_{3}$ such that $x(t) \geq m_{1}$ for $t$ large enough. Otherwise, we can select $m_{3}>0$ small enough and prove $x(t)<m_{3}$ does not hold for $t \geq 0$. By the condition of Theorem 2, let

$$
\sigma=\int_{n \tau}^{(n+1) \tau}\left(\frac{\beta}{\delta}\left(\tilde{z}(t)-\varepsilon_{1}\right)-d_{2}\right) d t>0
$$


We will prove that $x(t)<m_{3}$ cannot hold for $t \geq 0$. Otherwise,

$$
\begin{cases}\frac{d s(t)}{d t} \geq \lambda-\left(d_{1}+\beta m_{3}\right) s(t), & t \neq n \tau, t \neq(n+l) \tau \\ \Delta s(t)=\mu, & t=n \tau, \\ \Delta s(t)=-\mu_{1} s(t), & t=(n+l) \tau\end{cases}
$$

By Lemma 3, we have $s(t) \geq z(t)$ and $z(t) \rightarrow \tilde{z}(t), t \rightarrow \infty$, where $z(t)$ is the solution of

$$
\begin{cases}\frac{d z(t)}{d t}=\lambda-\left(d_{1}+\beta m_{3}\right) z(t), & t \neq n \tau, t \neq(n+l) \tau \\ \Delta z(t)=\mu, & t=n \tau, \\ \Delta s(t)=-\mu_{1} z(t), & t=(n+l) \tau\end{cases}
$$

and

$$
\tilde{z}(t)= \begin{cases}\frac{1}{\left(d_{1}+\beta m_{3}\right)}\left[\lambda-\left(\lambda-\left(d_{1}+\beta m_{3}\right) z^{*}\right) e^{-\left(d_{1}+\beta m_{3}\right)(t-n \tau)}\right], & t \in[n \tau,(n+l) \tau), \\ \frac{1}{\left(d_{1}+\beta m_{3}\right)}\left[\lambda-\left(\lambda-\left(d_{1}+\beta m_{3}\right) z^{* *}\right) e^{-\left(d_{1}+\beta m_{3}\right)(t-(n+l) \tau)}\right], & t \in[(n+l) \tau,(n+1) \tau) .\end{cases}
$$

Here $z^{*}$ and $z^{* *}$ are defined as

$$
z^{*}=\frac{\left.\lambda\left(1-\mu_{1} e^{-\left(d_{1}+\beta m_{3}\right)(1-l) \tau}\right)-\lambda\left(1-\mu_{1}\right) e^{-\left(d_{1}+\beta m_{3}\right) \tau}\right)+\left(d_{1}+\beta m_{3}\right) \mu}{\left(d_{1}+\beta m_{3}\right)\left[1-\left(1-\mu_{1}\right) e^{-\left(d_{1}+\beta m_{3}\right) \tau}\right]}
$$

and

$$
z^{* *}=\frac{1-\mu_{1}}{\left(d_{1}+\beta m_{3}\right)}\left[\lambda-\left(\lambda-\left(d_{1}+\beta m_{3}\right) z^{*}\right) e^{-\left(d_{1}+\beta m_{3}\right) l \tau}\right] .
$$

Therefore, there exists $T_{1}>0$ such that

$$
s(t) \geq z(t) \geq \tilde{z}(t)-\varepsilon_{1}
$$

and

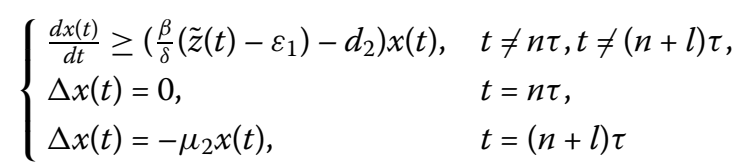

for $t \geq T_{1}$. Let $N_{1} \in N$ and $N_{1} \tau>T$. Integrating (4.12) on $(n \tau,(n+1) \tau]\left(n \geq N_{1}\right)$, we have

$$
\begin{aligned}
x((n+1) \tau) & \geq\left(1-\mu_{2}\right) x\left(n \tau^{+}\right) \exp \left(\int_{n \tau}^{(n+1) \tau}\left(\frac{\beta}{\delta}\left(\tilde{z}(t)-\varepsilon_{1}\right)-d_{2}\right) d t\right) \\
& =\left(1-\mu_{2}\right) x\left(n \tau^{+}\right) e^{\sigma} .
\end{aligned}
$$

Then $x\left(\left(N_{1}+K\right) \tau\right) \geq\left(1-\mu_{2}\right)^{K} x\left(N_{1} \tau^{+}\right) e^{K \sigma} \rightarrow \infty$, as $K \rightarrow \infty$, which is a contradiction to the boundedness of $x(t)$. Hence there exists $t_{1}>0$ such that $x(t) \geq m_{3}$. 
Thus, we can also obtain that $\frac{d s(t)}{d t} \geq \lambda-\left(d_{1}+\beta M\right) s(t)$, then the following comparatively impulsive differential equation is

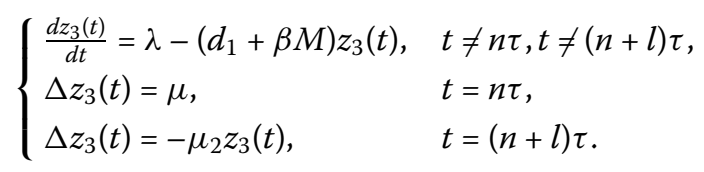

Similar to (3.7), we have

$$
\tilde{z}_{3}(t)= \begin{cases}\frac{1}{\left(d_{1}+\beta M\right)}\left[\lambda-\left(\lambda-\left(d_{1}+\beta M\right) z_{3}^{*}\right) e^{-\left(d_{1}+\beta M\right)(t-n \tau)}\right], & t \in[n \tau,(n+l) \tau), \\ \frac{1}{\left(d_{1}+\beta M\right)}\left[\lambda-\left(\lambda-\left(d_{1}+\beta M\right) z_{3}^{* *}\right) e^{-\left(d_{1}+\beta M\right)(t-(n+l) \tau)}\right], & t \in[(n+l) \tau,(n+1) \tau) .\end{cases}
$$

Here $z_{3}^{*}$ and $z_{3}^{* *}$ are defined as

$$
z_{3}^{*}=\frac{\left.\lambda\left(1-\mu_{1} e^{-\left(d_{1}+\beta m_{3}\right)(1-l) \tau}\right)-\lambda\left(1-\mu_{1}\right) e^{-\left(d_{1}+\beta m_{3}\right) \tau}\right)+\left(d_{1}+\beta m_{3}\right) \mu}{\left(d_{1}+\beta m_{3}\right)\left[1-\left(1-\mu_{1}\right) e^{-\left(d_{1}+\beta m_{3}\right) \tau}\right]}
$$

and

$$
z_{3}^{* *}=\frac{1-\mu_{1}}{\left(d_{1}+\beta m_{3}\right)}\left[\lambda-\left(\lambda-\left(d_{1}+\beta m_{3}\right) z^{*}\right) e^{-\left(d_{1}+\beta m_{3}\right) l \tau}\right] .
$$

For any $\varepsilon_{4}$ small enough, we obtain

$$
z_{3}(t)>\tilde{z}_{3}(t)-\varepsilon_{4}
$$

From the comparison theorem of impulsive differential equations, we have

$$
s(t)>z_{3}(t)>\tilde{z}_{3}(t)-\varepsilon_{4}>\left(z_{3}^{*}(t)+z_{3}^{* *}(t)\right)-\varepsilon_{4}=m_{4},
$$

i.e., $s(t)>m_{4}$. This completes the proof.

Remark Let

$$
F(\tau)=\ln \frac{1}{1-\mu_{2}}-\frac{\lambda \tau}{d_{1}}-\frac{\lambda-d_{1} s^{*}}{d_{1}^{2}}\left(1-e^{-d_{1} l \tau}\right)-\frac{\lambda-d_{1} s^{* *}}{d_{1}^{2}}\left(1-e^{-d_{1}(1-l) \tau}\right) e^{-d_{1} \tau}-d_{2} \tau .
$$

We easily know $F(0)=\frac{1}{\ln \left(1-\mu_{2}\right)}>0, F(\tau) \rightarrow-\infty$ as $\tau \rightarrow \infty$, and $F^{\prime \prime}(\tau)>0$, so $F(\tau)=0$ has a unique positive root, denoted by $\tau_{\max }$. From Theorems 1 and 2, we know $\tau_{\max }$ is a threshold. If $\tau<\tau_{\max }$, the microorganism-extinction periodic solution $(s(t), 0)$ of $(2.1)$ is globally asymptotically stable. If $\tau<\tau_{\max }$, system (2.1) is permanent.

\section{Discussion}

In this paper, according to the actual situation, we discussed the impulsive dredging at a fixed time. The global asymptotic stability of the microorganism-extinction periodic solution $(s(t), 0)$ of $(2.1)$ is discussed and the condition for the permanence of the system is also obtained. From Theorems 1 and 2, we can deduce that there must exist a threshold 
$\mu_{2}^{*}$. If $\mu_{2}>\mu_{2}^{*}$, the microorganism-extinction periodic solution $(s(t), 0)$ of $(2.1)$ is globally asymptotically stable. If $\mu_{2}<\mu_{2}^{*}$, system (2.1) is permanent. Our results indicate that the impulsive dredging and the period of impulsive dredging play an important role in water eutrophication management.

\section{Acknowledgements}

The authors are grateful to the anonymous referees for their excellent suggestions, which greatly improved the presentation of the paper.

\section{Funding}

The research was supported by the National Natural Science Foundation of China (11761019, 11361014), the Project of High Level Creative Talents in Guizhou Province (No. 20164035), and the Joint Project of Department of Commerce and Guizhou University of Finance and Economics (No. 2016SWBZD18).

\section{Competing interests}

The authors declare that they have no competing interests.

\section{Authors' contributions}

JJ corrected the manuscript, PL carried out the main part of this article, HF gave some suggestions for this article. All authors have read and approved the final manuscript.

\section{Publisher's Note}

Springer Nature remains neutral with regard to jurisdictional claims in published maps and institutional affiliations.

Received: 26 January 2018 Accepted: 13 August 2018 Published online: 22 October 2018

\section{References}

1. Wang, G., et al.: Causes, hazards and control measures of eutrophication in water body. J. Jilin Agric. Univ. 22 116-118 (2000)

2. Feng, Y:: Grey evaluation model of lake eutrophication and its application. J. Syst. Eng. Theory Pract. 15(2), 68-71 (1996)

3. Li, Q.: The grey dynamic model in lake TN. Application of water resources protection in the prediction of TP storage. 2, 23-26 (1998)

4. Li, Z., Dong, B.: A method for evaluating the degree of eutrophication of lake eutrophication based on the Euclid approach and the discussion of the method of evaluating the degree of closeness to the lake trophic state. J. Hamming Arid Environ. Monit. 1, 16-19 (1996)

5. Li, L., Dai, W.H.: Chaohu water rich Chinese soil and water conservation, pollution situation and control countermeasures. Soil Water Conserv. China 7, 55-57 (2009)

6. Yu, J., Liu, Y., et al.: Study on evaluation methods and dominant factors of eutrophication in Poyang Lake. J. Jiangxi Agric. Sci. 21(4), 125-128 (2009)

7. Jiao, J., Chen, L.: Study on extinction threshold of a single population model with impulsive input of environmental toxins in contaminated environment. J. Appl. Math. 22(1), 11-19 (2009)

8. Jiao, J., Chen, L.: Analysis of the Chemostat model with time delay and disturbance in the contaminated soil. J. Appl. Math. 23(4), 861-869 (2010)

9. Jiao, J., Bao, L.: Dynamic model of a single stage population structure with impulsive effects. J. Math. Pract. Cogn. 41(5), 162-166 (2011)

10. Lakshmikantham, V., Bainov, D.D., Simeonov, P.: Theory of Impulsive Differential Equation. World Scientific, Singapore (1989)

11. Bainov, D., Simeonov, P.: Impulsive Differential Equations: Periodic Solutions and Applications. Pitman Mongraphs Surveys Pure Appl. Math., vol. 66 (1993)

\section{Submit your manuscript to a SpringerOpen ${ }^{\circ}$ journal and benefit from:}

- Convenient online submission

- Rigorous peer review

- Open access: articles freely available online

- High visibility within the field

- Retaining the copyright to your article 